\title{
Paper Materials in Fashion Design Creative Applications
}

\author{
Haiyan Guo \\ Wuhan Institute of Clothing Textile University, Wuhan Hubei 430073 China , \\ E-mail:50154681@qq.com
}

Keywords : Paper clothing ; Environmental; Creative; Costume design

\begin{abstract}
Environmental , creativity is the development trend of fashion design. Will also be the direction of future development of clothing . Paper materials more and more fashion designers alike. Paper clothing in both environmental protection, creativity also meet people on the natural , comfortable, beautiful, healthy fashion needs in contemporary costume ideas promoted by eco-friendly clothing at a certain background paper has a broad space for development.
\end{abstract}

\section{Paper costume features}

Paper is one of the great inventions of China, is a modeling material with a variety of properties . Its low cost, wide range of uses , paper materials as a special material to be applied to clothing design is a relatively new attempt, paper cloth clothing costume charm is unmatched . Paper materials clothing has taken, environmental protection, design method is particularly good three-dimensional effect, rich colors, exotic textures, effects, and features a good stage performances . In recent years, the paper material on the performance and environmental performance by taking the support of science and technology, has been significantly improved. In Europe, designers DuPont developed the usual paper materials. In the application on paper garment more flexible than traditional fabrics in Asia, South Korean paper clothing forefront on wearability. South Korean paper blended silk or cotton, the fabric tactile comfortable and washable. Meanwhile, the Korean paper dry faster than other textiles , part of the performance even more than cotton and wool fabrics. Japanese Paper fabrics lightweight, breathable feel like linen. This material also has the effect of absorbing dust and odors . Not only that, these specially treated paper material processing technology still has the basic characteristics of the paper - biodegradable , low toxicity hazards, waste paper recycling reproduction rate. Recycled paper material to meet the requirements of the current development of recycling economy. And the rich resources of raw materials and paper, the current selection of raw materials waste paper or banana stalk stalk instead of wood to save the consumption of timber resources, greatly reducing the cost of production, the development of effective new renewable resources.

\section{Paper costume design}

Material properties of paper material determines the different design methods, the formation of the effect of different costumes . Flexible, absorbent paper material can be obtained by special visual effects tie-dye, printing method, textured yarn suitable for the production of laminated sheet material of the skirt shape. While combined with a variety of paper craft techniques to performance.

Paperwork .Paper materials can be used paper-cut method, by cutting, engraving, carving, or advanced laser technology process paper made hollow effect. As one of the folk art of the oldest Chinese handmade art, with its wealth of design themes, vivid rustic style form, as well as strong 
and rich colors emotion and renowned Chinese and foreign, and was favored by many fashion designers, the paper-cut lines, patterns and other elements in a variety of forms of distortion, portfolio, etc., applied to clothing design , unique artistic charm. ( See Figure 1 )

Origami. After the paper folded paper plane formed bump ups and downs to make three-dimensional effect . Origami is an ancient art of folk crafts, with a strong sense of three-dimensional space and special texture effects . Origami style in modern fashion design on the main outline of the overall three-dimensional shape and two forms of local decoration, brings a new aesthetic taste of clothing, and through a modern interpretation of the traditional process to achieve the purpose of promoting the national culture . ( See Figure 2 )

Fold .Soft and easy to use wrinkled paper material characteristics, processing into surface is not smooth texture effects, through the paper making process to produce the artistic effect garment folds, increasing the sense of space and layering of clothing. The costumes and design, folds as an important means of decoration and formal language, implies the creation of a combination of practical and aesthetic with good practical functionality and visual aesthetic , and thus derived from a rich and profound culture and arts connotation, is a combination of three-dimensional plane of the point . ( See Figure 3 )

Weaving.Because the human body is convex, a large area of stiff paper is difficult because there is no elasticity and body docile. But after the paper cut paper, paper is not bound by the force of the other piece of paper, which has more flexibility, plasticity, can show more costumes . ( See Figure 4 )

Flat collage.Collage is a method of the plane after a variety of colored paper cut engraved regroup, it requires hand firmly decisive, accurate. Plane is composed of tiles of different sizes with colored pieces of paper, master shape and color contrast and balance by moving, as well as the ability to use scissors and tape .

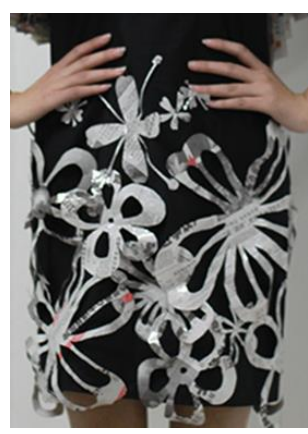

(Figure 1)



(Figure 2)

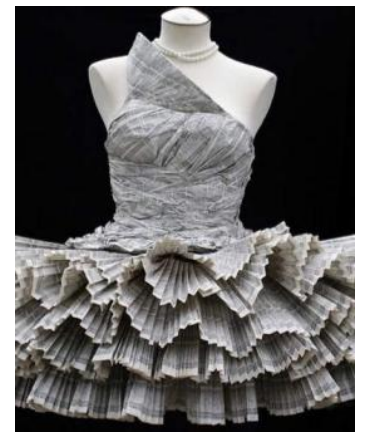

(Figure 3)

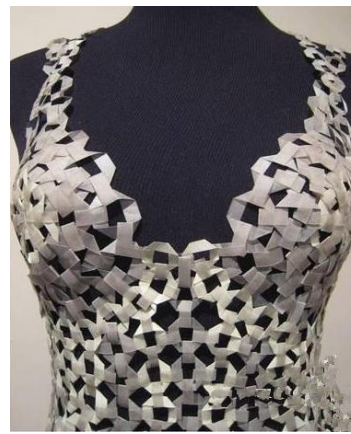

(Figure 4)

\section{Creative paper clothing}

Draping .Draping is the main form of paper clothing, it has the advantage in performance and a special three-dimensional modeling, we usually use the fabric to show wrinkles, the results are often unsatisfactory when folded, but if the use of paper to produce wrinkle effect is very good ( Figure 5,6 ) . Paper dimensional shape by folding paper, scissors cut, paste and other methods of three-dimensional design and has an ornamental and practical plastic arts . It summed up the image of an exaggeration, simple lines, strong decorative sense, this advantage clipping plane to catch .

Rich colors .Paper color is very rich, there are many types of paint colors there are many kinds of paper. Calendar calendar because it is the backing paper, pattern content above it determines its color different species, such as landscapes , still lifes , portraits , etc., so the color is rich , the basic 
color is yellow exposing colored kraft, wrapping paper and more is diverse, ever-changing background shading both have bright colors and elegant shading net surface, in addition to the effect of laser wrapping paper, you bring people to the science fiction era. In short nature of the colors are rich, the color of the paper is based on the extraction of natural colors, we can say inexhaustible.

Peculiar texture. So many kinds of paper texture of both rich and strange, a type of white wrapping paper such as cardboard, linerboard , kraft paper, corrugated paper, cardboard, brown paper, and so on . Printing paper such as coated paper, newsprint, newspapers and so on. Another industrial paper, office culture paper, tissue and specialty paper, such as decorative Watermark paper, pattern paper, and so on . Can directly use a lot of paper, some smooth as a mirror, and some hair shaggy, such as hemp and some rough, some smooth as silk. Also transform paper texture , texture and color can be made in different costumes .

\section{Paper materials apparel prospects}

In recent years, the paper clothing has appeared in various international fashion show . South Korea's Korean paper fashion show ( see Figure 7-8 ), Fashion Association and other statewide organizations and enterprises have made great achievements in the design of paper material applications in the international apparel market successfully launched by the various types of clothing garments made of paper material. If designed as a disposable wearing it, low production costs, materials and production process of environmental recovery, to provide a modern choice. More and more people come to realize and accept paper fashion. There are currently a number of famous international fashion designers paper paper fashion, dress development, application and promotion to make a certain contribution. The paper industry is still nascent in this fashion, it will continue to grow, more people being studied, more people are wearing . The current global "respect for nature , green consumption ," the increasing demands of environmental protection , countries are actively developing, manufacturing conducive to environmental protection, beneficial to human health green clothing, limiting the development of high energy consumption, high pollution industry, the development of energy environmentally friendly industries. Paper clothing applications just follow environmental requirements, but also for the world to make its own contribution to the cause of environmental protection .

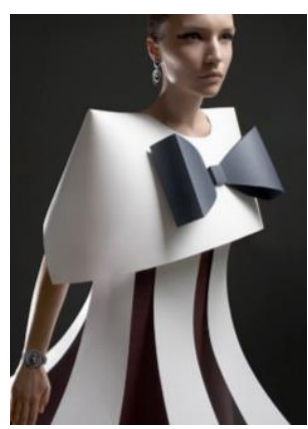

(Figure5)

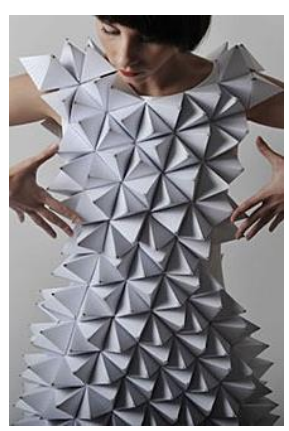

(Figure6)



(Figure 7)

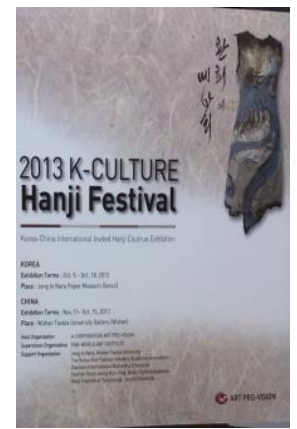

(Figure 8)

\section{References}

[1] Wu Liqun. Japan to develop textile paper line [J]. Tissue, 2003 (4): 47.

[2] Party Yuhong, Wang Zhijie, Lihong Kui. Textile and paper line of research paper [J]. China Pulp and Paper, 2006 (4): 56. 
[3] Bian Xiangyang: Clothing Art judgment [M], Shanghai, Donghua University Press, 2006. 164-165

[4] loess dragon; Introduction to Modern Fashion Culture [M], Shanghai, Donghua University Press, 2009. 200-201 